EP-139

\title{
A study for the expression of FAM83H, ZNF16, and FAM83H-related proteins in gallbladder cancer
}

\author{
Mirin LEE, Jae Do YANG, Sung Woo AHN, Hee Chul YU*
}

Department of General Surgery, Jeonbuk National University Hospital, Jeonju, Korea

Introduction: FAM83H is mostly known for its role in amelogenesis, however, recent reports suggest FAM83H might be involved in tumorigenesis. A search of the public database shows a significant association between FAM83H and ZNF16 in various carcinomas including biliary tract cancer. Although the studies of FAM83H in gallbladder cancer are limited, we inferred gallbladder cancer has similar traits with other biliary cancers.

Methods: We evaluated the clinicopathological significance of the immunohistochemical expression of FAM83H and ZNF16 in 105 gallbladder cancer patients.

Results: The expression of FAM83H and ZNF16 were significantly associated with each other. In univariate analysis, individual, and co-expression pattern of FAM83H and ZNF16 was significantly associated with shorter overall survival (OS) and relapse-free survival (RFS) of gallbladder cancer patients: nuclear expression of FAM83H (OS; $p<0.001)$, cytoplasmic expression of FAM83H (OS; $p<$ 0.01 ), nuclear expression of ZNF16 (OS; $p<0.001$ ), cytoplasmic expression of ZNF16 (OS; $p=0.032$ ). In multivariate analysis, nuclear expression of FAM83H (OS; $p<0.001)$ and the co-expression pattern of nuclear FAM83H and ZNF16 (OS; $\mathrm{p}<0.001)$ were independent indicators of shorter survival of gallbladder patients.

Conclusions: These results suggest that FAM83H and ZNF16 might be involved in the progression of gallbladder cancer, and their expression might be used as novel progression indicator for GB cancer patients. 